\title{
Globe
}

Revue internationale d'études québécoises

\section{Élisabeth Gallat-Morin et Jean-Pierre Pinson : La vie musicale en Nouvelle-France. Sillery, Septentrion, 2003}

\section{Pascal Blanchet}

Volume 9, numéro 1, 2006

URI : https://id.erudit.org/iderudit/1000809ar

DOI : https://doi.org/10.7202/1000809ar

Aller au sommaire du numéro

Éditeur(s)

Globe, Revue internationale d'études québécoises

ISSN

1481-5869 (imprimé)

1923-8231 (numérique)

Découvrir la revue

Citer ce compte rendu

Blanchet, P. (2006). Compte rendu de [Élisabeth Gallat-Morin et Jean-Pierre Pinson : La vie musicale en Nouvelle-France. Sillery, Septentrion, 2003]. Globe, 9(1), 288-291. https://doi.org/10.7202/1000809ar d'utilisation que vous pouvez consulter en ligne.

https://apropos.erudit.org/fr/usagers/politique-dutilisation/ 
Il est évident que cette anthologie représente la somme d'un énorme travail effectué par les deux chercheures que sont Dumont et Toupin. Toutefois, l'occultation de textes plus littéraires, comme la prose de Nicole Brossard, ou de pièces de théâtre, telle Les fées ont soif de Denise Boucher, peut étonner. En effet, ces manifestations culturelles ont tout autant contribué à l'essor de la pensée féministe et à son développement social que les textes des militantes des autres disciplines. En outre, si le nombre d'extraits est impressionnant, il aurait été pertinent d'en présenter certains dans leur totalité afin de faire parler les textes dans leur plénitude rhétorique. Finalement, une question persiste : pourquoi ne pas avoir considéré les nouveaux discours féministes qui circulent depuis 1985 jusqu'en 2000, alors que certaines féministes radicales se font encore entendre et qu'une nouvelle génération de féministes s'applique à dresser un portrait actualisé de la condition féminine? Cela étant dit, Micheline Dumont et Louise Toupin, avec leur Anthologie de la pensée féministe au Québec, ont accompli une tâche inégalée dans le domaine, et leur ouvrage fera certainement le bonheur des chercheur(e)s et historien(ne)s des idées au Québec.

\author{
Cristian Micu et \\ Marie-France Raymond-Dufour \\ Université de Toronto
}

\title{
Élisabeth Gallat-Morin et Jean-Pierre Pinson
}

La vie musicale en Nouvelle-France.

Sillery, Septentrion, 2003.

À force de vivre dans un monde sursaturé de musique, on en viendrait presque à oublier qu'il n'en a pas toujours été ainsi. On ne s'étonne plus de tant de choix, que ce soit en téléchargeant une chanson sur Internet, en furetant dans le bac d'un disquaire où se retrouvent soldées les vedettes d'hier, ou même en s'impatientant de subir dans le métro à la fois la musique de haut-parleurs déficients et la complainte du musicien ambulant - quand ce n'est pas le baladeur du voisin! Parmi les nombreux attraits que comporte la lecture de La vie musicale en Nouvelle-France, qu'il nous soit permis de mentionner une rafraîchissante remise en perspective, qui nous amène à méditer sur le lien 
qu'entretenaient nos ancêtres avec la musique. Bien sûr, il n'est question ici que de "quelques arpents de neige", mais on peut extrapoler : la situation * des artisans, des notaires, des maîtres d'école, des marchands, des militaires, des bourgeois ou des aristocrates, des administrateurs et des responsables politiques * (p. 19) de la Nouvelle-France par rapport à la musique différait-elle tant, au fond, de celle de leurs contreparties européennes? Hors des grandes villes, où trouver de la musique? Comment en "faire ", à moins d'aller à l'église (où l'on n'est même pas sûr de trouver un orgue) ? Quelle place occupe la musique dans la vie d'un être humain quand il doit lutter quotidiennement pour sa survie et qu'il n'a, au fond, que sa voix pour chanter ou ses pieds pour marquer le rythme? Il faut en tout cas que ce désir de musique soit bien grand pour avoir survécu à l'existence difficile qui était le lot des habitants de l'Amérique française - ceux qui venaient d'arriver, mais aussi ceux qui y étaient déjà.

Il faut aussi que le pouvoir de la musique soit bien grand et que la fascination qu'elle exerce soit puissante pour expliquer l'empressement des autorités religieuses à en faire entendre à ses ouailles. À ce propos, on n'est pas surpris de constater que la musique religieuse occupe la moitié du livre qui fait plus de cinq cents pages. On sait l'importance qu'a eue la religion en nos contrées, et ce, jusqu'à il n'y a pas si longtemps. La musique religieuse est celle qui a laissé le plus de traces : les auteurs ont pu consulter plusieurs documents musicaux de bonne qualité, en plus de nombreux récits de contemporains sur la façon de faire la musique religieuse, comme en témoignent les annexes, constituées de mandements d'évêques, de statuts et de règlements à l'usage des curés et de leurs chantres. En fait, la surprise viendrait plutôt du fait qu'on ait trouvé tant de choses à dire sur la "musique en société *: "Dans un pays où le clergé est défavorable au thêâtre et à l'opéra, c'est à l'église et dans les processions que s'expriment la ferveur populaire et le "goût du spectacle" "(p. 18). En l'absence de lieux consacrés officiellement à l'écoute de la musique profane, il n'en reste que ses expressions les plus spontanées, soit les chansons, les fêtes, les bals. Toutes ces manifestations laissent peu de traces, aussi les auteurs ont-ils eu soin d'aller jusqu'à dépouiller des inventaires de biens, actes notariés qui laissent parfois deviner la présence d'instruments de musique ou de partitions dans les foyers. Cette recherche très poussée, qui tient à la fois de l'archẹologie et de l'enquête policière, nous permet d'apprendre quantité de faits étonnants au sujet de la vie musicale en Nouvelle-France. 


\section{REVUE INTERNATIONALE D'ÉTUDES QUÉBÉCOISES}

Car la musique est étonnamment présente sous le Régime français, depuis Le théâtre de Neptune, sorte d'opéra aquatique présenté "sur les flots du Port Royal " le 14 novembre 1606 (deux ans avant la fondation de Québec), jusqu'aux folles dépenses de l'intendant Bigot, grand organisateur de soupers, de bals et de concerts vers la fin du régime activités qui suscitent la réprobation du marquis de Montcalm, qui s'en plaint dans son Journal.

Déjà, dans les années 1698 à 1701, Claude-Charles Bacqueville de la Potherie avait noté que les Canadiennes avaient " beaucoup de disposition à danser "et Pierre de Sales Laterrière, écrit à la fin du xviı" siècle : "Jamais je n'ai connu nation aimant plus à danser que les Canadiens" (p. 292).

Maints exemples savoureux de cette passion ponctuent la deuxième partie du livre, consacrée à la musique profane : on y décrit de graves notables ou de riches marchands qui, ayant chaussé leurs " pantoufles de bal "spécialement achetées pour l'occasion, " coulent "le menuet jusqu'à en perdre leur perruque, ou bien de jeunes gens fringants qui se trémoussent au son d'une contredanse appelée Le Rbinocéros. Pour s'adonner aux joies du rythme, on peut bien sûr faire appel à des "violons de hasard ", mais ce n'est pas nécessaire: "[...] l'absence d'instruments n'empêchait pas de danser, puisque l'on pouvait danser en chantant, comme l'indique le titre d'un livre que possédait François Chalet, de la Compagnie des Indes, Les menuets chantants * (p. 302).

Le chant, d'ailleurs, n'est pas en reste. Le fondateur de Montréal, Paul Chomedey de Maisonneuve, se servait-il de son habileté à jouer du luth pour accompagner quelque jolie chanteuse ? On l'ignore, mais on sait qu'on chantait des cantates et des cantatilles jusque dans les couvents - sur des sujets religieux, bien sûr. De nombreux citoyens avaient en leur possession des partitions d'opéras, qui étaient alors des œuvres populaires, à commencer par les tragédies lyriques de Lully (dont un commentateur, cité dans le livre, affirme que " toutes les cuisinières de France fredonnaient ses airs. (p. 308)), mais aussi les pastorales de Joseph Bodin de Boismortier et les opéras de Michel-Richard Delalande. Ce dernier, surintendant de la musique de Louis XIV, a pour petit-neveu un certain François Mion, aventurier et musicien, qui traversera l'Atlantique au xviII siècle, emportant avec lüi des partitions de son parent. Les airs d'opéra se voient d'ailleurs fréquemment transformés : on garde la 


\section{RECENSIONS}

mélodie et on change les paroles pour louer, à son gré, le bon Dieu ou le bon vin. Mais la musique peut aussi mener loin : ainsi, un peintre rebelle est condamné à être exposé sur la place du marché, le cou dans un carcan, pour avoir composé une chanson satirique. Plus sagement, on peut aussi se réunir entre musiciens pour * concerter ", autrement dit faire de la musique ensemble pour le plaisir, sans public.

Mais outre ces aspects pittoresques, qui sont comme autant de petits romans (et dont nos scénaristes de cinéma devraient bien s'inspirer), La vie musicale en Nouvelle-France est avant tout un ouvrage de très haute érudition, la somme de tous les savoirs disponibles sur le sujet au moment de sa parution. La réputation des auteurs n'est plus à faire : Élisabeth Gallat-Morin et Jean-Pierre Pinson œuvrent dans le domaine depuis plus de vingt ans; c'est autant de temps qu'il en a fallu pour rassembler toute cette information. De plus, ils se sont entourés des plus grands spécialistes en la matière : Paul-André Dubois pour la musique religieuse chez les Amérindiens, Conrad Laforte pour les musiques de tradition orale (dont le chapitre sur les chansons de canotiers constitue aussi un épisode passionnant), Erich Schwandt pour le petit motet dans les communautés religieuses féminines et François Filiatrault, qui s'est chargé de la recherche iconographique, laquelle est généreuse sinon abondante, du moins pour ce type d'ouvrage.

Il serait vain de prétendre que la lecture d'un livre aussi savant soit vraiment aisée. On imagine mal un lecteur, même averti, le dévorant dans son intégralité. Il sera surtout utile aux chercheurs et aux étudiants, qui trouveront là tout ce qui existe sur le sujet, en plus de leur inspirer un nombre considérable de nouvelles recherches possibles. En fait, pour juger adéquatement de ce livre, il faudrait être soi-même un spécialiste de l'envergure des auteurs... Tout au plus pourra-t-on reprocher à cette publication d'un sérieux impressionnant - et parfois étrangement jubilatoire - de ne pas avoir osé défier la tradition : on rêve d'une réédition dans laquelle les notes seraient disponibles au bas des pages et non en fin de volume, ce qui soumet le lecteur à une gymnastique des plus fastidieuses. Mais ce léger bémol ne doit diminuer en rien les grandes qualités du livre, la première étant de faire réfléchir sur la place de la musique dans nos vies, la seconde étant de nous donner - peut-être - envie de chanter.

Pascal Blanchet Université de Montréal 